International Journal of Wavelets, Multiresolution and Information Processing

(C) World Scientific Publishing Company

\title{
An Analysis Method for Sampling in Shift-Invariant Spaces
}

\author{
Stefan Ericsson and Niklas Grip \\ Department of Mathematics \\ Luleå University of Technology, SE-97187 Luleå, Sweden. \\ E-mail: \{grip,sen\}@sm.luth.se \\ Received (02 012003$)$ \\ Revised (2901 2004) \\ Communicated by (xxxxxxxxxx)
}

\begin{abstract}
A subspace $V$ of $L_{2}(\mathbb{R})$ is called shift-invariant if it is the closed linear span of integershifted copies of a single function.

As a complement to classical analysis techniques for sampling in such spaces, we propose a method which is based on a simple interpolation estimate of a certain coefficient mapping. Then we use this method for deriving both new results and relatively simple proofs of some previously known results. Among these are both some results of rather general nature and some more specialized results for B-spline wavelets.

The main problem under study is to find a shift $x_{0}$ and an upper bound $\delta$ such that any function $f \in V$ can be reconstructed from a sequence of sample values $\left(f\left(x_{0}+k+\delta_{k}\right)\right)_{k \in \mathbb{Z}}$, either when all $\delta_{k}=0$ or in the irregular sampling case with an upper bound $\sup _{k}\left|\delta_{k}\right|<\delta$.
\end{abstract}

Keywords: shift-invariant space; biorthogonal wavelets; irregular sampling; regular sampling; B-spline wavelet; interpolation; reproducing kernel Hilbert space.

AMS Subject Classification: 94A20, 41A15, 41A30, 42C15, 42C40, 46E22, 46N99, 47A15, 47B32, 47B37, 93C57.

\section{Introduction}

Most modern signal processing methods can be described as data processing of sample values $f\left(\lambda_{k}\right)$ of an analyzed function $f$ with some non-discrete domain, such as $\mathbb{R}$. Even if the sampling points are meant to be regularly distributed, say, $\lambda_{k}=x_{0}+k$ with $k \in \mathbb{Z}$, practical restrictions, such as imperfect clocks (for other examples, see $\left.{ }^{2}\right)$ may introduce a jitter error $\delta_{k} \stackrel{\text { def }}{=} \lambda_{k}-\left(x_{0}+k\right)$. Two important questions arise: For "how large" $\delta_{k}$ can $f$ be reconstructed from the sample values $f\left(\lambda_{k}\right)$, and is there a practically useful method for doing this? We will mainly target the first of these questions. To be more precise, using basic definitions and properties of frames and Riesz bases (described with further references in pages 71-72 of the second author's doctoral thesis ${ }^{8}$ ), we propose a method for analysis of sampling in so-called shift-invariant spaces $V \subseteq L_{2}(\mathbb{R})$ with the following properties: 
$V$ is the $L_{2}$-closed span of a Riesz basis $\left(\varphi_{k}\right)_{k \in \mathbb{Z}} \stackrel{\text { def }}{=}(\varphi(\cdot-k))_{k \in \mathbb{Z}}$

with $\varphi$ belonging to the amalgam space $W\left(L_{\infty}, l_{1}\right)$, that is

$$
\|\varphi\|_{W\left(L_{\infty}, l_{1}\right)}<\infty, \quad \text { where }\|\varphi\|_{W\left(L_{\infty}, l_{p}\right)} \stackrel{\text { def }}{=}\left(\sum_{k \in \mathbb{Z}} \sup _{x \in[0,1)}|\varphi(x+k)|^{p}\right)^{\frac{1}{p}}
$$

for $1 \leq p \leq \infty$. In a typical application, $\varphi$ can be, for example, the scaling function of some wavelet basis or the impulse response of some measurement device.

For $\varphi \in W\left(L_{\infty}, l_{2}\right)$, we show in Lemma 2.1 that $V$ possesses a family of functions $q_{x}$ with the characterizing property

$$
\forall f \in V, \quad f(x)=\left\langle f, q_{x}\right\rangle, \quad \text { a.e. } x \in \mathbb{R} .
$$

We denote the functions $q_{x}$ reproducing kernels and call the corresponding $V$ a reproducing kernel Hilbert space. For such spaces, there are several papers (such as $415 \mid 141152324$ ) with sampling results of the following type (or less general): Given $\varphi$, can we find a translation $x_{0}$ and an upper bound $\delta$ such that

$$
f=\sum_{k \in \mathbb{Z}} f\left(\lambda_{k}\right) s_{\lambda_{k}}, \quad \forall f \in V,
$$

for some sequence $\left(s_{\lambda_{k}}\right)$ in $V$, and either

$$
\lambda_{k}=x_{0}+k+\delta_{k} \quad \text { and } \quad \sup _{k \in \mathbb{Z}}\left|\delta_{k}\right|<\delta \quad \text { (irregular sampling), }
$$

or all $\delta_{k}=0$ (regular sampling)?

For numerical stability (in the sense of a finite condition number) of the analysis mapping $f \mapsto\left(f\left(\lambda_{k}\right)\right),\left(s_{\lambda_{k}}\right)$ must at least be a frame for $V$ (see page 17 of ${ }^{8}$ ). In fact, if $\left(q_{\lambda_{k}}\right)$ is a frame for $V$ with dual frame $\left(\widetilde{q_{\lambda_{k}}}\right)$, then the reconstruction formula (1.3a) holds with $s_{\lambda_{k}}=\widetilde{q_{\lambda_{k}}}$, since then the sample values $f\left(\lambda_{k}\right)$ coincide with the minimum $l_{2}$-norm ${ }^{825}$ frame series expansion coefficients $\left\langle f, q_{\lambda_{k}}\right\rangle$. Finally, for $\left(q_{\lambda_{k}}\right)$ to also have a numerically stable synthesis mapping $\mathrm{T}_{q}:\left(f\left(\lambda_{k}\right)\right) \mapsto f$, $\left(q_{\lambda_{k}}\right)$ must be a Riesz basis for $V$ (see page 20 of ${ }^{8}$ ).

The Riesz basis property is not necessarily an advantage, however. Without it, the stability of $\mathrm{T}_{q}$ (as a mapping from $l_{2}$ to $V$ ) is lost because the sample values $f\left(\lambda_{k}\right)$ are restricted to a strict (minimum $l_{2}$-norm) subspace $U$ of $l_{2}$. However, with $\mathrm{T}_{q}$ restricted to this space we get the numerical stability back and in communications applications with the transmitted data stored in the coefficients, an orthonormal projection of the sample values to $U$ in the receiver will provide stability against noise and other additive errors. (See also Remark 3.3.)

In the remaining paper, we mainly consider Riesz basis reproducing kernels. Frame reproducing kernels and extensions to (weighted) $L_{p}\left(\mathbb{R}^{d}\right)$-spaces are used in ${ }^{1 / 2 / 10}$ for the study of irregular sampling that is not caused by jitter errors.

Example 1.1. The probably oldest and most well-known example is the Shan- 
non sampling theorem, which follows when $\varphi(t)=\sin (\pi \omega t) /(\pi t) \stackrel{\text { def }}{=} \operatorname{sinc}_{\omega}(t)$ and, consequently, $V=\left\{f \in L_{2} \mid \operatorname{supp} \widehat{f} \subseteq[-\omega / 2, \omega / 2]\right\}$ (see, e.g., ${ }^{71423}$ ). In fact, $V$ is a reproducing kernel Hilbert space with reproducing kernel $q_{x}=\operatorname{sinc}_{\omega}(\cdot-x)$. This follows from an application of the Plancherel theorem to the inverse Fourier transform of $\widehat{f}$ : For a.e. $x \in \mathbb{R}$,

$$
f(x)=\int_{\mathbb{R}} \widehat{f}(\xi) e^{i 2 \pi \xi x} \mathrm{~d} \xi=\int_{\mathbb{R}} \widehat{f}(\xi) \overline{\chi_{\left[-\frac{\omega}{2}, \frac{\omega}{2}\right]}(\xi) e^{-i 2 \pi \xi x}} \mathrm{~d} \xi=\int_{\mathbb{R}} f(t) \operatorname{sinc}_{\omega}(t-x) \mathrm{d} t,
$$

that is, $q_{x}$ satisfies (1.2). Since the Fourier transform is an isometric mapping of $V$ onto $L_{2}([-\omega, \omega])$, it follows that $\left(\operatorname{sinc}_{\omega}\left(\cdot-\lambda_{k}\right)\right)_{k}$ is a Riesz basis for $V$ if and only if $\left(e^{i 2 \pi \lambda_{k} x}\right)_{k}$ is a Riesz basis for $L_{2}([-\omega, \omega])$. Thus the irregular sampling problem for the Shannon wavelet is equivalent to the Kadec 1/4-theorem, which tells that if $\sup _{k}\left|\lambda_{k}-k\right|<\frac{1}{4}$, then $\left(e^{i 2 \pi \lambda_{k} x}\right)_{k}$ is a Riesz basis for $V$ (see, e.g., ${ }^{92125}$ ).

After some mathematical preliminaries in Section 2, the main results follow in Section 3, where we derive a method for analysis of problems related to reconstruction formulas $f=\sum_{k \in \mathbb{Z}} f\left(\lambda_{k}\right) \widetilde{q_{\lambda_{k}}}$. Crucial to our approach is that we investigate the equivalent problem of whether a certain doubly infinite matrix defines an invertible mapping of $l_{2}$ onto $l_{2}$. This matrix consists of sample values of $\varphi$, so we can use simple interpolation estimates to obtain invertibility conditions of the type $\sum_{k \neq 0}\left|\varphi\left(x_{0}+k+\delta_{k}\right)\right|<\left|\varphi\left(x_{0}+\delta_{0}\right)\right|$. From this we get an immediate suggestion of how to choose $x_{0}$, as well as some new results and relatively simple proofs of previously known results. For example, we show that if the reconstruction formula (1.3) holds when all $\delta_{k}=0$, then it holds also for some $\delta>0$. In particular, we use this to prove the existence of such an irregular sampling reconstruction formula for every B-spline wavelet. We do also show, for example, that if $\left(q_{x_{0}+k}\right)$ is a frame, then it is a Riesz basis.

Notation: Throughout the paper we consider functions defined on $\mathbb{R}$ and sequences with index set $\mathbb{Z}$. Thus we often use shorthand notation like $\left(a_{k}\right)_{k},\left(a_{k}\right)$ or $L_{2}$ instead of $\left(a_{k}\right)_{k \in \mathbb{Z}}$ and $L_{2}(\mathbb{R})$.

\section{Preliminaries}

Since every $f \in L_{2}$ is an equivalence class of functions that pairwise coincide almost everywhere, notions like continuity and pointwise evaluation make sense only when applied to fixed representatives $f^{*} \in f$ of $f$ (for example in (2.6)). Thus we will need a rule for which representative to choose: Note therefore that $\varphi$ always will be given by a predefined representative $\varphi^{*}$, which we assume (without loss of generality) to be defined on all of $\mathbb{R}$. Thus, from now on and for all $f \in V$ we will always consider either $f$ or the representative

$$
f^{*}(x) \stackrel{\text { def }}{=} \sum_{k \in \mathbb{Z}}\left\langle f, \widetilde{\varphi}_{k}\right\rangle \varphi_{k}^{*}(x), \quad \forall x \in \mathbb{R} .
$$


Under the decay assumption $\varphi \in W\left(L_{\infty}, l_{2}\right)$, the Cauchy-Schwarz inequality ensure pointwise convergence to $f^{*}$, which coincide with the corresponding $L_{2}$-limit almost everywhere (by Theorem 3.12 in $\underline{16}$ ), so $f^{*}$ is indeed a representative of $f$. It will usually be apparent from the context whether we refer to $f$ or $f^{*}$, so for brevity we usually write $f$ as shorthand notation for $f^{*}$.

Remark 2.1. Lemmas 2.1 and 2.3 is a collection and generalization of results first proven by Walter and Liu ${ }^{1423}$ with similar but not identical proofs. We consider the generalization to discontinuous $\varphi$ especially important since in several applications (e.g. with $\varphi$ being the impulse response of some measurement device) $\varphi$ may very well be far from continuous. Although not needed in most of our proofs, we note that in a typical sampling application, $\varphi$ should be continuous on the set $\left.\cup_{k \in \mathbb{Z}}\right] x_{0}+$ $k-\delta, x_{0}+k+\delta[$, since this, by (2.6) below, gives continuity in all potential sampling points. For different extensions of Lemma 2.1 (and more) to $L_{p}\left(\mathbb{R}^{d}\right)$ - and weighted $L_{p}\left(\mathbb{R}^{d}\right)$-spaces, see 11210 .

Lemma 2.1 (Reproducing kernel). For $\varphi \in W\left(L_{\infty}, l_{2}\right)$, suppose that $\varphi_{k}=$ $\varphi(\cdot-k)$ and $\widetilde{\varphi}_{k}=\widetilde{\varphi}(\cdot-k)$ are dual Riesz bases for their $L_{2}$-closed span $V$. Then

$\forall f \in V$ are continuous on the set $\mathfrak{C}=\left\{x \mid \varphi_{k}\right.$ is continuous at $\left.x, \forall k \in \mathbb{Z}\right\}$.

Moreover, for all $x \in \mathbb{R}$, unconditional convergence holds for

$$
q_{x} \stackrel{\text { def }}{=} \sum_{k \in \mathbb{Z}} \overline{\varphi_{k}(x)} \widetilde{\varphi}_{k} \quad \text { both in } L_{2}(\mathbb{R}) \text { and uniformly on } \mathbb{R} \text {. }
$$

It follows that $q_{x}$ is a reproducing kernel, that is, (with notation from (2.5))

$$
f^{*}(x)=\left\langle f, q_{x}\right\rangle, \quad \forall f \in V, \quad \forall x \in \mathbb{R} .
$$

It follows also that if $f \in V$ and $\left(f_{k}\right)_{k}$ is a sequence in $V$, then

$$
f_{n} \rightarrow f \text { in } V \quad \Rightarrow \quad f_{n} \rightarrow f \text { uniformly on } \mathbb{R} .
$$

In addition, if $\varphi \in W\left(L_{\infty}, l_{1}\right) \subseteq W\left(L_{\infty}, l_{2}\right)$, then also $\widetilde{\varphi} \in W\left(L_{\infty}, l_{1}\right)$.

Proof. It follows from the definition of $\left(\widetilde{\varphi}_{k}\right)_{k \in \mathbb{Z}}$ (showed with a repeated use of the (generalized) Parseval identity in the proof of Theorem 2 in 5 ) that there is a $c_{\varphi} \in \mathbb{R}_{+}$such that for all $x \in \mathbb{R}, \sum_{k \in \mathbb{Z}}|\widetilde{\varphi}(x-k)|^{2} \leq c_{\varphi}^{2} \sum_{k \in \mathbb{Z}}|\varphi(x-k)|^{2}$. Thus for $\varphi \in W\left(L_{\infty}, l_{2}\right)$ and $K \subseteq Z$, the Cauchy-Schwarz inequality gives

$$
\begin{aligned}
\left|\sum_{k \in K} \overline{\varphi_{k}(x)} \widetilde{\varphi}_{k}(\xi)\right| & \leq c_{\varphi}\left(\sum_{k \in K}|\varphi(x-k)|^{2}\right)^{\frac{1}{2}}\left(\sum_{k \in \mathbb{Z}}|\varphi(\xi-k)|^{2}\right)^{\frac{1}{2}} \\
& \leq c_{\varphi}\left(\sum_{k \in \mathbb{Z}} \sup _{a \in[k, k+1)}|\varphi(x-a)|^{2}\right)^{\frac{1}{2}}\left(\sum_{k \in \mathbb{Z}} \sup _{b \in[k, k+1)}|\varphi(\xi-b)|^{2}\right)^{\frac{1}{2}} \\
& \leq c_{\varphi} \sup _{x \in[0,1)}\|\varphi(x-\cdot)\|_{W\left(L_{\infty}, l_{2}\right)}^{2} \leq 2 c_{\varphi}\|\varphi\|_{W\left(L_{\infty}, l_{2}\right)}^{2}<\infty .
\end{aligned}
$$


Hence, by (2.10) and the argument following (2.5), (2.7) converges pointwise to a limit that coincides almost everywhere with the $L_{2}$-limit and this convergence is unconditional since $\left(\widetilde{\varphi}_{k}\right)$ is a Riesz basis for $V \stackrel{8}{8}^{[}$Next, for finite $K \subset \mathbb{Z}$, (2.11), the Lebesgue dominated convergence theorem and (2.5) show that for all $f \in V$

$$
\left\langle f, q_{x}\right\rangle=\sum_{k \in \mathbb{Z}} \int_{\mathbb{R}} f(\xi) \overline{\widetilde{\varphi}_{k}(\xi) \overline{\varphi_{k}(x)}} \mathrm{d} \xi=\sum_{k \in \mathbb{Z}}\left\langle f, \widetilde{\varphi}_{k}\right\rangle \varphi_{k}(x) \stackrel{\text { def }}{=} f^{*}(x), \quad \forall x \in \mathbb{R},
$$

with convergence both pointwise and in $L_{2}$. This proves (2.8). Next, by (2.7) and the Riesz basis definition ${ }^{8},\left\|q_{x}\right\| \leq B\left(\sum_{k \in \mathbb{Z}}|\varphi(x-k)|^{2}\right)^{1 / 2} \leq$ $B \sup _{x \in[0,1)}\|\varphi(x-\cdot)\|_{W\left(L_{\infty}, l_{2}\right)} \leq \sqrt{2} B\|\varphi\|_{W\left(L_{\infty}, l_{2}\right)} \stackrel{\text { def }}{=} c^{\prime}<\infty$ for some $B \in \mathbb{R}_{+}$. Hence, by (2.12),

$$
\left|f(x)-f_{n}(x)\right|=\left|\left\langle f-f_{n}, q_{x}\right\rangle\right| \leq\left\|q_{x}\right\|\left\|f-f_{n}\right\| \leq c^{\prime}\left\|f-f_{n}\right\|, \quad \forall f, f_{n} \in V,
$$

which proves $(2.9),(2.6)$ and the uniform convergence in (2.7). Finally, that $\varphi \in$ $W\left(L_{\infty}, l_{1}\right)$ implies $\widetilde{\varphi} \in W\left(L_{\infty}, l_{1}\right)$ is proved by Aldroubi and Gröchenig. ${ }^{2}$

Remark 2.2. The explicit formula (2.7), requires knowledge of $\widetilde{\varphi}$, which sometimes can be cumbersome to compute. However, in the remaining paper, we will only need Lemma 2.1 for checking the existence of the reproducing kernel (which is unique in $V$ by the Riesz representation theorem).

Example 2.1 (Spline wavelets). A B-spline scaling function $\varphi=B_{n}$ of degree $n$ is defined as follows: Let $B_{0}=\chi_{[0,1]}$ and $B_{n}=\chi_{[0,1]} * B_{n-1}$ for $n \in \mathbb{Z}_{+}$. By Lemma 2.1, the associated $V$ is a reproducing kernel Hilbert space.

For separable Hilbert spaces $\mathcal{H}$ and $\mathcal{H}_{i}$, let $\mathcal{G}(\mathcal{H}) \stackrel{\text { def }}{=} \mathcal{G}(\mathcal{H}, \mathcal{H})$, with $\mathcal{G}\left(\mathcal{H}_{1}, \mathcal{H}_{2}\right)$ denoting the set of linear, bounded and invertible mappings of $\mathcal{H}_{1}$ onto $\mathcal{H}_{2}$ (hence, by the open mapping theorem, the inverse mapping is also bounded). In next lemma, we use the fact ${ }^{825}$ that a sequence $r \stackrel{\text { def }}{=}\left(r_{k}\right)$ is a Riesz basis for $\mathcal{H}$ if and only if the synthesis mapping $\mathrm{T}_{r}:\left(c_{k}\right) \mapsto \sum_{k \in \mathbb{Z}} c_{k} r_{k}$ is a well-defined function in $\mathcal{G}\left(l_{2}, \mathcal{H}\right)$.

Lemma 2.2 (Matrix representation). Suppose that $\left(\varphi_{k}\right)$ is a Riesz basis for a separable Hilbert space $\mathcal{H}$ and denote with $r=\left(r_{k}\right)$ an arbitrary sequence in $\mathcal{H}$. Let $L$ and $\Phi$ be the linear mapping and the doubly infinite matrix defined by

$$
L \varphi_{k} \stackrel{\text { def }}{=} r_{k} \quad \text { and } \quad(\Phi)_{k, n} \stackrel{\text { def }}{=}\left\langle r_{n}, \varphi_{k}\right\rangle .
$$

Then the following statements are equivalent:

$$
\left(r_{n}\right) \text { is a Riesz basis for } \mathcal{H} \text {. }
$$

$L$ can be uniquely extended to a function in $\mathcal{G}(\mathcal{H})$.

$$
\Phi \in \mathcal{G}\left(l_{2}\right) .
$$

Proof. It is well-known (see pages 176 and 26 in 25$)$ that $(2.14 \mathrm{a}) \Leftrightarrow(2.14 \mathrm{~b})$. 


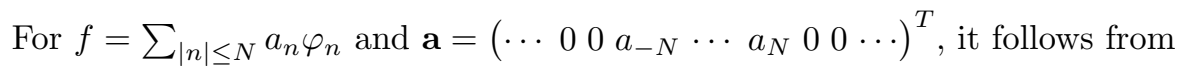

$$
\begin{aligned}
L f & =\sum_{k \in \mathbb{Z}}\left\langle L f, \varphi_{k}\right\rangle \widetilde{\varphi}_{k}=\sum_{k \in \mathbb{Z}} \sum_{|n| \leq N} a_{n}\left\langle r_{n}, \varphi_{k}\right\rangle \widetilde{\varphi}_{k}=\sum_{k \in \mathbb{Z}}(\Phi \mathbf{a})_{k} \widetilde{\varphi}_{k} \\
& =\mathrm{T}_{\widetilde{\varphi}} \Phi \mathbf{a}=\mathrm{T}_{\widetilde{\varphi}} \Phi \mathrm{T}_{\varphi}^{-1} f
\end{aligned}
$$

that $L=\mathrm{T}_{\tilde{\varphi}} \Phi \mathrm{T}_{\varphi}^{-1}$ on a dense subset of $\mathcal{H}$ and thus, since $\mathrm{T}_{\tilde{\varphi}}, \mathrm{T}_{\varphi} \in \mathcal{G}\left(l_{2}, \mathcal{H}\right)$, also on all of $\mathcal{H}$. Consequently, $(2.14 \mathrm{~b}) \Leftrightarrow(2.14 \mathrm{c})$ and the proof is complete.

For regular sampling, we will need the following lemma, which also can be generalized to necessary and sufficient conditions for a frame (or Riesz basis) reconstruction $f=\sum_{k} f(k) \widetilde{q_{k}}$ to hold.$^{26}$

Lemma 2.3. For $\varphi \in W\left(L_{\infty}, l_{1}\right), V$ and $q_{x}$ as in Lemma 2.1 and for arbitrary $x_{0} \in \mathbb{R}$, we define the multiplicative operator $\mathrm{M}: L_{2}([0,1]) \rightarrow L_{2}([0,1])$,

$$
\mathrm{M} f(\xi) \stackrel{\text { def }}{=} m(\xi) f(\xi), \quad \text { where } \quad m(\xi) \stackrel{\text { def }}{=} \sum_{l \in \mathbb{Z}} \overline{\varphi\left(x_{0}+l\right)} e^{-i 2 \pi l \xi} .
$$

Then, for some $A, B \in \mathbb{R}_{+}$, it follows that

$$
\left(q_{x_{0}+k}\right) \text { is a Riesz basis for } V \quad \Leftrightarrow \quad A \leq|m(\xi)| \leq B \text { for a.e. } \xi \in[0,1] .
$$

Proof. Since $\varphi \in W\left(L_{\infty}, l_{1}\right),\left(\varphi\left(x_{0}+l\right)\right)_{l \in \mathbb{Z}} \in l_{1} \subset l_{2}$. Thus $m$ is a welldefined and continuous (by uniform convergence) function in $L_{2}([0,1])$. Let $g(\xi) \stackrel{\text { def }}{=}$ $\sum_{|n| \leq N} a_{n} e^{i 2 \pi n \xi}$ and denote with $\Phi_{0}$ the matrix

$$
\left(\Phi_{0}\right)_{k, l} \stackrel{\text { def }}{=}\left\langle q_{x_{0}+l}, \varphi_{k}\right\rangle=\overline{\varphi_{k}\left(x_{0}+l\right)}=\overline{\varphi\left(x_{0}+l-k\right)}, \quad \forall k, l \in \mathbb{Z} .
$$

By proceeding as in the proof of Lemma 2.2 and using (2.8), we get

$$
\begin{aligned}
\mathrm{M} g(\xi) & =m(\xi) \sum_{|n| \leq N} a_{n} e^{i 2 \pi n \xi}=\sum_{l \in \mathbb{Z}} \overline{\varphi\left(x_{0}+l\right)} e^{-i 2 \pi l \xi} \sum_{|n| \leq N} a_{n} e^{i 2 \pi n \xi} \\
& =\sum_{|n| \leq N} \sum_{l \in \mathbb{Z}} \overline{\varphi\left(x_{0}+l\right)} a_{n} e^{i 2 \pi(n-l) \xi}=\sum_{|n| \leq N} \sum_{k \in \mathbb{Z}} a_{n} \overline{\varphi\left(x_{0}+n-k\right)} e^{i 2 \pi k \xi} \\
& =\sum_{k \in \mathbb{Z}} \sum_{|n| \leq N}\left\langle q_{x_{0}+n}, \varphi_{k}\right\rangle a_{n} e^{i 2 \pi k \xi}=\sum_{k \in \mathbb{Z}}\left(\Phi_{0} \mathbf{a}\right)_{k} e^{i 2 \pi k \xi}=\mathrm{F} \Phi_{0} \mathrm{~F}^{-1} g(\xi),
\end{aligned}
$$

where $\mathrm{F} \in \mathcal{G}\left(l_{2}, L_{2}([0,1])\right)$ is the Fourier series mapping $\left(c_{k}\right) \mapsto \sum_{k} c_{k} e^{i 2 \pi k}$. Since $\mathrm{F}$ is isometric, we conclude that $\mathrm{M}=\mathrm{F} \Phi_{0} \mathrm{~F}^{-1}$ on $L_{2}([0,1])$ and

$$
\mathrm{M} \in \mathcal{G}\left(L_{2}([0,1])\right) \quad \Leftrightarrow \quad \Phi_{0} \in \mathcal{G}\left(l_{2}\right) .
$$

Thus (2.17) follows from Lemma 2.2 with $r_{k}=q_{k}, A^{-1}=\left\|\mathrm{M}^{-1}\right\|$ and $B=\|\mathrm{M}\|$. 


\section{Main results}

We will now propose a technique for finding $x_{0}$ and $\delta$ such that

$$
f=\sum_{k \in \mathbb{Z}} f\left(\lambda_{k}\right) \widetilde{q_{\lambda_{k}}}, \quad \forall f \in V
$$

where $\left(q_{\lambda_{k}}\right)$ is a reproducing kernel Riesz basis for $V$,

$$
\lambda_{k}=x_{0}+k+\delta_{k} \quad \text { and } \quad \sup _{k \in \mathbb{Z}}\left|\delta_{k}\right|<\delta .
$$

Given that Lemma 2.1 applies, the reconstruction (3.20) holds if $\left(q_{\lambda_{k}}\right)$ is a Riesz basis, or equivalently (via Lemma 2.2 with $r_{k}=q_{\lambda_{k}}$ ), if $\Phi \in \mathcal{G}\left(l_{2}\right)$, where

$$
(\Phi)_{k, l} \stackrel{\text { def }}{=}\left\langle q_{\lambda_{l}}, \varphi_{k}\right\rangle=\overline{\varphi_{k}\left(\lambda_{l}\right)}=\overline{\varphi\left(\lambda_{l}-k\right)} .
$$

For any $\Lambda \in \mathcal{G}\left(l_{2}\right)$ (possibly depending on $\left(\delta_{k}\right)$ ), a sufficient condition for $(\Lambda \Phi$ and thus also) $\Phi$ to be invertible is that $\|\mathrm{I}-\Lambda \Phi\|<1$. For simplicity, we will choose a diagonal matrix $\Lambda$ with $k$ th entry $a_{k}$ and use a simple interpolation estimate of the norm. (Other choices, such as $\Lambda=\Phi_{0}^{-1}$ may however be useful for some particular choices of $\varphi$.) This gives Theorem 3.1, which contain the key results of this paper: Condition (i) is the simplest one. The simplicity is in itself an important strength, which sometimes makes (i) easy to use and intuitively appealing (see Remark 3.1 and Theorem 3.2). Condition (i) is a special case of the more technical condition (ii), which follows from setting all $a_{k}=a \neq 0$ in $\Lambda$. Condition (iii), finally, follows by setting $a_{k}=1 /(\Phi)_{k, k}$. The main reason for this choice of $\Lambda$ is that since $\Lambda \Phi$ has ones on the diagonal, it seems likely that $\|\mathrm{I}-\Lambda \Phi\|<\|\mathrm{I}-\Phi\|$. A careful comparison of conditions (ii) and (iii) would be interesting, but is out of the scope of this article. We will see, however, that condition (iii) gives larger upper bounds $\delta$ when applied to B-splines in Example 3.1.

Theorem 3.1. With $\varphi \in W\left(L_{\infty}, l_{1}\right)$ and notation as in Lemma 2.1 and (3.20b), set

$$
\alpha \stackrel{\text { def }}{=} \inf _{\left|x-x_{0}\right| \leq \delta} \varphi(x) \quad \text { and } \quad \beta_{k} \stackrel{\text { def }}{=} \sup _{|x| \leq \delta}\left|\varphi\left(x_{0}+k+x\right)\right| .
$$

(i) Then (3.20a) holds if $\sum_{k \neq 0} \beta_{k}<\alpha$, that is, if

$$
\sum_{k \neq 0} \sup _{|x| \leq \delta}\left|\varphi\left(x_{0}+k+x\right)\right|<\inf _{\left|x-x_{0}\right|<\delta} \varphi(x) .
$$

(ii) A more general condition (which includes (i) as a special case) is that $\alpha>0$ and

$$
\sup _{|x|<\delta}\left(\sum_{j \neq 0}\left|a \varphi\left(x_{0}+j+x\right)\right|+\left|1-a \varphi\left(x_{0}+x\right)\right|\right)\left(a \sum_{k \neq 0} \beta_{k}+\gamma(a)\right)<1
$$


$8 \quad$ Stefan Ericsson and Niklas Grip

for some $a \in \mathbb{R} \backslash\{0\}$ and with

$$
\gamma(a) \stackrel{\text { def }}{=} \max \left(1-\alpha a, \beta_{0} a-1\right)=\left\{\begin{array}{ll}
1-\alpha a & \text { if } a \leq \frac{2}{\alpha+\beta_{0}} \\
\beta_{0} a-1 & \text { if } a>\frac{2}{\alpha+\beta_{0}}
\end{array} .\right.
$$

If (3.24) holds for some $a \neq 0$, then the smallest possible left-hand side is obtained for some $a \in\left[1 / \beta_{0}, 1 / \alpha\right]$ (depending on $\varphi$ and $\delta$ ).

(iii) Yet another sufficient condition for (3.20a) to hold is that

$$
\sup _{l \in \mathbb{Z}} \frac{\sum_{j \neq 0}\left|\varphi\left(x_{0}+j+\delta_{l}\right)\right|}{\left|\varphi\left(x_{0}+\delta_{l}\right)\right|} \sup _{l \in \mathbb{Z}} \frac{\sum_{j \neq 0}\left|\varphi\left(x_{0}+j+\delta_{j+l}\right)\right|}{\left|\varphi\left(x_{0}+\delta_{l}\right)\right|}<1 .
$$

Proof. As explained after (3.21), we need to find $x_{0}$ and $\delta$ such that

$$
\sup _{k \in \mathbb{Z}}\left|\delta_{k}\right|<\delta \quad \Longrightarrow \quad\|\mathrm{I}-\Lambda \Phi\|_{\mathcal{B}\left(l_{2}, l_{2}\right)}<1
$$

for some diagonal matrix $\Lambda$ with $k$ th entry $a_{k}, 1 /\left\|\Lambda^{-1}\right\|=\inf _{k}\left|a_{k}\right|>0$ and $\|\Lambda\|=$ $\sup _{k}\left|a_{k}\right|<\infty$. We estimate the norm with a special case of the Riesz interpolation theorem that was first proved by Schur ${ }^{3 / 18}$ and is based on the observation that according to the Cauchy-Schwarz inequality,

$$
\begin{aligned}
\|\mathrm{A} x\|^{2} & =\sum_{i}\left|\sum_{j} a_{i, j} x_{j}\right|^{2} \leq \sum_{i}\left(\sum_{j}\left|a_{i, j}\right|\right) \sum_{j}\left|a_{i, j}\right|\left|x_{j}\right|^{2} \\
& \leq \sup _{j} \sum_{i}\left|a_{i, j}\right| \cdot \sup _{i} \sum_{j}\left|a_{i, j}\right| \cdot \sum_{j}\left|x_{j}\right|^{2} .
\end{aligned}
$$

Consequently,

$$
\|\cdot\|_{\mathcal{B}\left(l_{2}, l_{2}\right)}^{2} \leq\|\cdot\|_{\mathcal{B}\left(l_{1}, l_{1}\right)}\|\cdot\|_{\mathcal{B}\left(l_{\infty}, l_{\infty}\right)}
$$

where $\mathcal{B}\left(\mathcal{B}_{1}, \mathcal{B}_{2}\right)$ denotes the set of linear bounded operators $f: \mathcal{B}_{1} \rightarrow \mathcal{B}_{2}$. In particular, it follows from (3.20b) and (3.21) that

$$
\begin{aligned}
&\|\mathrm{I}-\Lambda \Phi\|_{\mathcal{B}\left(l_{2}, l_{2}\right)}^{2} \leq \sup _{l} A_{l}\left(\Lambda,\left(\delta_{k}\right)\right) \cdot \sup _{l} B_{l}\left(\Lambda,\left(\delta_{k}\right)\right) \\
& \stackrel{\text { def }}{=} \sup _{l}\left(\sum_{k \neq l}\left|a_{k} \varphi\left(x_{0}+(l-k)+\delta_{l}\right)\right|+\left|1-a_{l} \varphi\left(x_{0}+\delta_{l}\right)\right|\right) \times \\
& \quad \times \sup _{l}\left(\sum_{k \neq l}\left|a_{l} \varphi\left(x_{0}+(k-l)+\delta_{k}\right)\right|+\left|1-a_{l} \varphi\left(x_{0}+\delta_{l}\right)\right|\right) .
\end{aligned}
$$

(iii): Set all $a_{k}=a \in \mathbb{R} \backslash\{0\}$ in (3.29). In this special case, we will now explicitly 
compute smallest upper bounds $A(a)$ and $B(a)$ such that

$$
\begin{aligned}
\sup _{\sup \left|\delta_{k}\right|<\delta} & \left(\sup _{l} A_{l}\left(\Lambda,\left(\delta_{k}\right)\right) \cdot \sup _{l} B_{l}\left(\Lambda,\left(\delta_{k}\right)\right)\right) \\
= & \sup _{\sup \left|\delta_{k}\right|<\delta} \sup _{l} A_{l}\left(\Lambda,\left(\delta_{k}\right)\right) \cdot \sup _{\sup \left|\delta_{k}\right|<\delta} \sup _{l} B_{l}\left(\Lambda,\left(\delta_{k}\right)\right) \stackrel{\text { def }}{=} A(a) B(a) .
\end{aligned}
$$

In fact, for any $\varepsilon>0$ we see from (3.25) that we can explicitly choose $\left(\delta_{k}\right)$ and $l$ such that $\left|B_{l}\left(\Lambda,\left(\delta_{k}\right)\right)-B(a)\right|<\varepsilon / 2$ for the (smallest) upper bound

$$
B(a)=a \sum_{k \neq 0} \beta_{k}+\sup _{|x| \leq \delta}\left|1-a \varphi\left(x_{0}+x\right)\right|=a \sum_{k \neq 0} \beta_{k}+\gamma(a)
$$

of the second factor in (3.29). Then we can pick $L \neq 0$ so that $\beta_{L}<\varepsilon / 2$ and change $\delta_{L}$ so that

$$
\left|A_{L}\left(\Lambda,\left(\delta_{k}\right)\right)-A(a)\right|<\varepsilon .
$$

After this change of $\delta_{L}$, we still have $\left|B_{l}\left(\Lambda,\left(\delta_{k}\right)\right)-B(a)\right|<\varepsilon$ for some $l$. This proves that the right-hand side of (3.29) has the smallest upper bound given by $(\overline{3.30})$ and (3.31). Consequently, if (3.24) holds, then so does the invertibility condition $\|\mathrm{I}-\Lambda \Phi\|<1$. Thus it remains to choose an $a$ that is likely to give a small left-hand side $A(a) B(a)$ in (3.24): Note from (3.24) and (3.25) that $B(a)$ is a piecewise linear function that is growing with increasing $|a|$ when $a<0$ or $a>2 /\left(\alpha+\beta_{0}\right)$. Thus

$$
B(a) \rightarrow \inf _{x} B(x) \text { when } a \rightarrow 0 \text { or when } a \rightarrow 2 /\left(\alpha+\beta_{0}\right) .
$$

Similarly, in (3.32), $A_{L}(a)$ grows with increasing $|a|$ when $a<0$ or $a>1 / \varphi\left(x_{0}+\delta_{L}\right)$. Here $\delta_{L}$ depends on $\delta$ and $\varphi$, so in general, we only know that $\alpha \leq \varphi\left(x_{0}+\delta_{L}\right) \leq \beta_{0}$. Hence, for a minimum $A(a)$, one should let $a \rightarrow 0$ or pick some $\left.a \in] 1 / \beta_{0}, 1 / \alpha\right]$. The case $a \rightarrow 0$ is, however, uninteresting, since it follows from (3.24) that

$$
B(a) \geq A(a) \geq \sup _{|x| \leq \delta}\left|1-a \varphi\left(x_{0}+x\right)\right| \geq\left|1-a \beta_{0}\right| \rightarrow 1 \quad \text { as } a \rightarrow 0 .
$$

Thus, if $A(a) B(a)<1$ for some $a$, then $A(a) B(a)$ is minimal for some $a \in$ ] $\left.1 / \beta_{0}, 1 / \alpha\right]$. This concludes the proof of (ii).

(i): Since $B(a) \geq A(a)$, the condition (3.24) follows if $B(a)<1$. Moreover, it follows from (3.31) and the definition (3.25) of $\gamma(a)$ that for $\left.a \in] 0,2 /\left(\alpha+\beta_{0}\right)\right]$, $B(a)<1$ if and only if $\sum_{k \neq 0} \beta_{k}<\alpha$. This proves (i).

(iii): Contrary to (ii), we do now use our freedom to let $a_{k}$ depend on $\left(\delta_{n}\right)$. Thereby we lose some simplicity (we can no longer first compute the suprema and then minimize the resulting expression), but a clever choice of $a_{k} \mathrm{~s}$ might make $\|\mathrm{I}-\Lambda \Phi\|$ smaller and allow for a larger $\delta$. Thus we insert $a_{k}=1 /(\Phi)_{k, k}$, in (3.29), which then becomes equal to (3.26). This concludes the proof.

Remark 3.1. It is intuitively clear from (3.23) that $x_{0}$ should be chosen so that $\varphi$ is as large as possible "near $x_{0}$ " and small "near $x_{0}+k$ ", $k \in \mathbb{Z} \backslash\{0\}$. Hence it 
seems likely that for "reasonably fast decaying" $\varphi$, a nearly optimal $\delta$ occurs for some $x_{0} \approx x_{\max }$, such that (assuming the supremum to be attained)

$$
\left|\varphi\left(x_{\max }\right)\right|=\sup _{x \in \mathbb{R}}|\varphi(x)|<\infty .
$$

This importance of $x_{0}$ was first discovered by Janssen ${ }^{[12}$, who showed that a translation $x_{0}$ is required to get sampling theorems of the type (3.20) for B-spline scaling functions of degree 2. This observation can be used as a rule of thumb, for example, in wavelet prefiltering, which can be described as a multiplication of input sample values with $\left(\Phi^{*}\right)^{-1}$, where $\Phi^{*}$ is the conjugate transpose of $\Phi$. In a forthcoming paper ${ }^{6}$, we compare different approximations of $\left(\Phi^{*}\right)^{-1}$ for a large number of wavelets and find that $x_{0}=x_{\max }$ usually gives reasonably small approximation errors, but for that particular application, an experimentally found optimal $x_{\max }$ turned out to give clear further improvements.

Remark 3.2. One way to further sharpen the conditions of Theorem 3.1 is to replace the condition $\|I-\Lambda \Phi\|<1$ with the sharper sufficient (but still not necessary) invertibility condition that (for some given $\Lambda$ )

$$
\left\|(\mathrm{I}-\Lambda \Phi)^{n}\right\|<1 \quad \text { for some } n \in \mathbb{Z}_{+},
$$

which also can be shown to give a Neumann series expansion of $(\Lambda \Phi)^{-1}$. The difficulty in this last approach is that it can be complicated to compute $(\mathrm{I}-\Lambda \Phi)^{n}$ for large $n$, except for some rare special case (such as in the proof of Theorem 3.3).

Another immediate generalization (allowing for complex-valued $\varphi$ but not needed here) is to replace the assumption $\alpha>0$ in (ii) with $\sup _{\left|x-x_{0}\right| \leq \delta}\left|1-a \varphi\left(x_{0}+x\right)\right|<1$ and an appropriately modified function $\gamma$.

Proposition $3.1\left(\left(\boldsymbol{q}_{x_{0}+\boldsymbol{k}}\right)\right.$ frame $\Rightarrow\left(\boldsymbol{q}_{x_{0}+\boldsymbol{k}}\right)$ Riesz basis $)$. If $\varphi \in W\left(L_{\infty}, l_{1}\right)$ and if $\left(q_{x_{0}+k}\right)_{k \in \mathbb{Z}}$ is a frame, then it is a Riesz basis.

Proof. Note first that since $\varphi \in W\left(L_{\infty}, l_{1}\right)$, the Schur interpolation estimate (3.28) implies that $\Phi \in \mathcal{B}\left(l_{2}, l_{2}\right)$. This guarantees the existence of the Hilbert-adjoint operator $\Phi^{*} \in \mathcal{B}\left(l_{2}, l_{2}\right)$ (which is represented by the conjugate transpose of the matrix $\Phi$ ). Thus Lemma 2.1 gives that for any $f=\mathrm{T}_{\varphi} \mathbf{a}=\sum_{k \in \mathbb{Z}} a_{k} \varphi_{k} \in V$,

$\left(\Phi^{*} \mathbf{a}\right)_{k}=\sum_{l \in \mathbb{Z}}\left(\Phi^{*}\right)_{k, l} a_{l}=\sum_{l \in \mathbb{Z}} \varphi\left(\lambda_{k}-l\right) a_{l}=\sum_{l \in \mathbb{Z}} \varphi\left(\lambda_{k}-l\right)\left\langle f, \widetilde{\varphi}_{l}\right\rangle=f\left(\lambda_{k}\right)=\left\langle f, q_{\lambda_{k}}\right\rangle$.

By inserting (3.37) in the frame definition (3.39) and recalling that the synthesis mapping $\mathrm{T}_{\varphi}: \mathbf{a} \mapsto f=\sum_{k \in \mathbb{Z}} a_{k} \varphi_{k}$ is boundedly invertible, we get that

$$
\begin{aligned}
\left(q_{\lambda_{k}}\right)_{k \in \mathbb{Z}} \text { is a frame for } V & \Leftrightarrow \\
\Leftrightarrow \quad \exists A, B \in \mathbb{R}_{+}: & A\|f\|^{2} \leq \sum_{k \in \mathbb{Z}}\left|\left\langle f, q_{\lambda_{k}}\right\rangle\right|^{2} \leq B\|f\|^{2}, \quad \forall f \in \mathcal{H}
\end{aligned}
$$




$$
\Leftrightarrow \quad \exists A^{\prime}, B^{\prime} \in \mathbb{R}_{+}: \quad A^{\prime}\|\mathbf{a}\|^{2} \leq\left\|\Phi^{*} \mathbf{a}\right\|_{l_{2}}^{2} \leq B^{\prime}\|\mathbf{a}\|^{2}, \quad \forall \mathbf{a} \in l_{2} .
$$

Now suppose that $\left(q_{k}\right)_{k \in \mathbb{Z}}$ is a frame for $V$. For the corresponding regular sampling matrix $\Phi_{0}$ (defined in (2.18) ), it follows from a substitution $m=l-n+k$ that

$$
\begin{aligned}
\left(\Phi_{0}^{*} \Phi_{0}\right)_{k, l} & =\sum_{m} \overline{\left(\Phi_{0}\right)_{m, k}}\left(\Phi_{0}\right)_{m, l}=\sum_{m} \varphi\left(x_{0}+k-m\right) \overline{\varphi\left(x_{0}+l-m\right)} \\
& =\sum_{n} \overline{\varphi\left(x_{0}+n-k\right)} \varphi\left(x_{0}+n-l\right)=\sum_{n}\left(\Phi_{0}\right)_{k, n}\left(\Phi_{0}^{*}\right)_{l, n}=\left(\Phi_{0} \Phi_{0}^{*}\right)_{k, l},
\end{aligned}
$$

that is, $\Phi_{0}$ is normal, so it follows from (3.40) that

$$
A^{\prime}\|\mathbf{a}\|^{2} \leq\left\|\Phi_{0}^{*} \mathbf{a}\right\|^{2}=\left\|\Phi_{0} \mathbf{a}\right\|^{2}, \quad \forall \mathbf{a} \in l_{2} .
$$

Since $\Phi_{0}$ is normal, $A^{\prime}\|\mathbf{a}\|^{2} \leq\left\|\Phi_{0} \mathbf{a}\right\|^{2}$ if and only if $\Phi_{0} \in \mathcal{G}\left(l_{2}\right)$ (see Theorem 12.12 in 17), so (3.41) and Lemma 2.2 (with $\left.r_{k}=q_{x_{0}+k}\right)$ gives that $\left(q_{x_{0}+k}\right)$ is a Riesz basis.

Remark 3.3. As first noted in a different context in the Introduction, we see again from (3.37) and (3.40) that the sample value sequences are restricted to some (minimum $l_{2}$-norm) subset $U \subseteq l_{2}$ of coefficients, for which $\Phi^{*} \in \mathcal{G}\left(l_{2}, U\right)$. We also know from Lemma 2.2 that $U$ is a strict subspace if and only if $\left(q_{\lambda_{k}}\right)$ is a frame but not a Riesz basis. We see also that in practical applications, the previously discussed stability against perturbed sample values via orthogonal projection on $U$ can be performed, e.g., by applying the pseudoinverse ${ }^{19}$ of $\Phi^{*}$ in (3.37) to compute an estimate of a from the sample values $f\left(\lambda_{k}\right)$. A closer study of this is out of the scope of this paper. Note, however, that non-Riesz basis frames also have the advantage of an inherent redundancy, which makes it possible to reconstruct the sampled signal also if some sample values are lost.2

Theorem 3.2 (Regular sampling $\Rightarrow$ irregular sampling). Suppose that $\varphi \in$ $W\left(L_{\infty}, l_{1}\right)$ generates a shift-invariant space $V$ such that the reproducing kernel Riesz basis reconstruction formula (3.20) holds when all $\delta_{k}=0$. If $\varphi$ is continuous on the set $I \stackrel{\text { def }}{=} \cup_{k \in \mathbb{Z}}\left[x_{0}+k-\delta, x_{0}+k+\delta\right]$, then (3.20) holds also for some $\delta>0$.

Proof. According to Lemma 2.2 and our assumptions, $\Phi_{0} \in \mathcal{G}\left(l_{2}\right)$, where $\Phi_{0}$ is the regular sampling matrix $(2.18)$. We will now show that the mapping

$$
l_{\infty} \ni\left(\delta_{k}\right) \mapsto \Phi \in \mathcal{B}\left(l_{2}, l_{2}\right),
$$

is continuous. Since the set of invertible elements in a Banach algebra is open, this continuity immediately implies that $\Phi \in \mathcal{G}\left(l_{2}\right)$ (and thus (3.20a) holds) also for all $\delta$ in some neighborhood of 0 . Thus it only remains to prove the claimed continuity:

For arbitrary $\delta \in(0,1 / 2]$ and $L<\delta$, assume that all $\left|\delta_{k}\right| \leq L$. Then for 
$g_{k}(x) \stackrel{\text { def }}{=} \varphi(x)-\varphi\left(x+\delta_{k}\right)$, the Schur interpolation (3.28) gives that

$$
\begin{aligned}
\left\|\Phi_{0}-\Phi\right\| & \leq \sup _{l}\left(\sum_{j \in \mathbb{Z}}\left|g_{l}\left(x_{0}+j\right)\right|\right) \sup _{l}\left(\sum_{j \in \mathbb{Z}}\left|g_{j+l}\left(x_{0}+j\right)\right|\right) \stackrel{\text { def }}{=} F_{1} F_{2} \\
& \leq \sup _{\left|\delta_{k}\right| \leq L} F_{1} \cdot \sup _{\left|\delta_{k}\right| \leq L} F_{2} \leq \sup _{\left|\delta_{k}\right| \leq L} F_{2}^{2} .
\end{aligned}
$$

For $\varepsilon>0$ and $N \in \mathbb{Z}_{+}$such that $R_{N} \stackrel{\text { def }}{=} \sum_{|j|>N} \sup _{|x|<\frac{1}{2}}\left|\varphi\left(x_{0}+j+x\right)\right|<\frac{\varepsilon}{4}$,

$$
\delta<\frac{1}{2} \Rightarrow F_{2} \leq \sup _{l} \sum_{|j| \leq N}\left|g_{j+l}\left(x_{0}+j\right)\right|+2 R_{N}<\sup _{l} \sum_{|j| \leq N}\left|g_{j+l}\left(x_{0}+j\right)\right|+\frac{\varepsilon}{2} .
$$

Since $\varphi$ is uniformly continuous on $\left[-\left(x_{0}+N+1 / 2\right), x_{0}+N+1 / 2\right] \cap I$, we can choose $\delta$ such that $\left|g_{j+l}\left(x_{0}+j\right)\right|<\varepsilon /(2(2 N+1))$ in the given summation interval. Hence $\left\|\Phi_{0}-\Phi\right\|<\varepsilon^{2}$ and $(\overline{3.42})$ is continuous. This concludes the proof.

Remark 3.4. Theorem 3.2 is a generalization of results first proved by Liu and Walter ${ }^{14}$, who worked in a slightly less general setting and with the more restrictive conditions $\left\|\left(\delta_{k}\right)\right\|_{l_{2}}<\delta$ or all $\delta_{k}=\delta$. Chen, Itoh and Shiki $\frac{\sqrt{4}}{\text { generalized these result }}$ to biorthogonal bases and $\left\|\left(\delta_{k}\right)\right\|_{l_{\infty}}<\delta$, but with a longer and more technical proof than ours. Sun and Zhou ${ }^{22}$ also prove a related result.

Corollary 3.1. For every B-spline scaling function, there is an irregular sampling reproducing kernel Riesz basis reconstruction formula of the type (3.20).

Proof. By Theorem 3.2, we only need to prove the reconstruction formula (3.20) for $\lambda_{k}=x_{0}+k$, which is trivial for the Haar scaling function $\varphi=B_{0}$. Thus, let $\varphi=B_{n}$ with $n \geq 1$, and with $B_{n}$ defined in Example 2.1. The reconstruction formula holds if $\left(q_{x_{0}+k}\right)$ is a Riesz basis or equivalently, by (2.17), if

$$
0<A \leq|m(\xi)| \leq B<\infty, \quad \text { a.e. } \xi \in[0,1], \quad m(\xi) \stackrel{\text { def }}{=} \sum_{l \in \mathbb{Z}} \overline{B_{n}\left(x_{0}+l\right)} e^{-i 2 \pi l \xi} .
$$

With $x_{\max }$ defined in (3.35), it follows immediately from the definition of $B_{n}$ that $x_{\max }=(n+1) / 2$ and that

$$
\widehat{B_{n}}(\xi)=\left(\int_{0}^{1} e^{-i 2 \pi x \xi} \mathrm{d} x\right)^{n+1}=\left(\frac{1-e^{-i 2 \pi \xi}}{i 2 \pi \xi}\right)^{n+1}=\left(e^{-i \pi \xi} \frac{\sin (\pi \xi)}{\pi \xi}\right)^{n+1} .
$$

We claim (and prove below) that for $x_{0}=x_{\max }$, the Poisson summation formula 
gives

$$
\begin{aligned}
m(\xi) & \stackrel{\text { def }}{=} \sum_{k \in \mathbb{Z}} B_{n}\left(x_{0}+k\right) e^{-i 2 \pi k \xi}=\sum_{k \in \mathbb{Z}} e^{i 2 \pi x_{0}(\xi-k)} \widehat{B_{n}}(\xi-k) \\
& =\sum_{k \in \mathbb{Z}} e^{i \pi(n+1)(\xi-k)}\left(e^{-i \pi(\xi-k)} \frac{\sin (\pi(\xi-k))}{\pi(\xi-k)}\right)^{n+1} \\
& =\sin ^{n+1}(\pi \xi) \sum_{k \in \mathbb{Z}}\left(\frac{(-1)^{k}}{\pi(\xi-k)}\right)^{n+1} .
\end{aligned}
$$

Since $B_{n}$ has compact support, it follows from (3.46) that $m(0)>0, m$ is continuous, 1-periodic and that $m(\xi)=m(-\xi)$. Thus, in order to prove the existence of bounds $0<A \leq|m(\xi)| \leq B<\infty$, it suffices to show that $m(\xi) \neq 0$ for $0<\xi \leq 1 / 2$. For odd $n$ this is clearly true. For even $n$, the series in (3.47) equals $\sum_{l \in \mathbb{Z}}\left(b_{2 l-1}+b_{2 l}\right)$, where

$$
\begin{aligned}
b_{2 l-1}+b_{2 l} & \stackrel{\text { def }}{=}\left(\frac{(-1)^{2 l-1}}{\pi(\xi-(2 l-1))}\right)^{n+1}+\left(\frac{(-1)^{2 l}}{\pi(\xi-2 l)}\right)^{n+1} \\
& =\frac{1}{(\pi(2 l-1-\xi))^{n+1}}-\frac{1}{(\pi(2 l-\xi))^{n+1}}>0, \quad \forall l \in \mathbb{Z}, \forall \xi \in\left(0, \frac{1}{2}\right] .
\end{aligned}
$$

Now it only remains to confirm the second equality in (3.46). One way to do this is to apply the Poisson summation formula (e.g., as in 13 and with $f(x)=$ $\left.B_{n}\left(x+x_{0}\right) e^{-i 2 \pi \xi x}\right)$. Perhaps more natural in this case is to simply compute the Fourier series coefficients of the right-hand side series (using the fact that the series is 1-periodic, uniformly convergent and has uniformly bounded partial sums).

Proposition 3.2. For the B-splines $B_{n}$ defined in Example 2.1, conditions (iii) and (iii) of Theorem 3.1 can be rewritten in the following simpler form: For $n \in$ $\mathbb{N}$ and $\varphi=B_{n}$, we have an irregular sampling reconstruction $f=\sum_{k} f\left(\lambda_{k}\right) \widetilde{q_{\lambda_{k}}}$ (as described in (3.20) ) if $\delta$ satisfy one of the following inequalities (which are polynomial inequalities in $\delta$ when $\delta \leq 1 / 2$ ):

$$
\begin{aligned}
\text { (ii) : } & p(\delta) \cdot\left(p(\delta)+\int_{\frac{1}{2}-\delta}^{\frac{1}{2}+\delta} B_{n}\left(x+\frac{n+1}{2}\right) d x\right)<1, \\
& \text { where } p(\delta) \stackrel{\text { def }}{=} 2-2 B_{n}\left(x_{0}+\delta\right) . \\
\text { (iii) : } \quad & 1-2 B_{n}\left(x+x_{0}\right)+\left(1-B_{n}\left(x+x_{0}\right)\right) \int_{\frac{1}{2}-\delta}^{\frac{1}{2}+\delta} B_{n}\left(x+x_{0}\right) d x<0
\end{aligned}
$$

Proof. It follows from our definition of $B_{n}$ (see, e.g., Section 4.2 in ${ }^{11}$ ) that for $x_{0}=(n+1) / 2, B_{n}\left(\cdot+x_{0}\right)$ is positive, symmetric, decreasing on $\mathbb{R}_{+}, \operatorname{supp} B_{n}=$ $[0, n+1]$ and

$$
\sum_{k \in \mathbb{Z}} B_{n}(x+k)=1, \quad \forall x \in \mathbb{R} .
$$


The last property also holds for orthonormal wavelets (by the well-known property (Theorem 1.7 in ${ }^{11}$ ) $\int_{\mathbb{R}} \varphi_{j, k}(x) \mathrm{d} x=\widehat{\varphi}(0)=1$ ), but the others are special properties of B-spline wavelets.

(ii): According to condition (ii) of Theorem 3.1, we need to find $\delta$ such that $A(a) B(a)<1$, where

$$
A(a) B(a)=\sup _{|x|<\delta}\left(\sum_{j \neq 0}\left|a \varphi\left(x_{0}+j+x\right)\right|+\left|1-a \varphi\left(x_{0}+x\right)\right|\right)\left(a \sum_{k \neq 0} \beta_{k}+\gamma(a)\right) .
$$

We compute $A$ by using the inverse Fourier transform of $\widehat{B_{n}}$ (given by (3.45)), setting $x_{0}=(n+1) / 2$, and noticing that the odd part of the integrand vanishes:

$$
\begin{aligned}
B_{n}\left(x+x_{0}\right) & =\int_{\mathbb{R}}\left(e^{-i \pi \xi} \frac{\sin (\pi \xi)}{\pi \xi}\right)^{n+1} e^{i 2 \pi\left(x+x_{0}\right) \xi} \mathrm{d} \xi \\
& =\int_{\mathbb{R}}\left(\frac{\sin (\pi \xi)}{\pi \xi}\right)^{n+1} \cos (2 \pi x \xi) \mathrm{d} \xi .
\end{aligned}
$$

Now set $a=1$ and use (3.48):

$$
A(1)=\sup _{|x|<\delta}\left(1-B_{n}\left(x_{0}+x\right)+\sum_{j \neq 0}\left|B_{n}\left(x_{0}+j+x\right)\right|\right)=2-2 B_{n}\left(x_{0}+\delta\right) \stackrel{\text { def }}{=} p(\delta) .
$$

Next, note that $a=1<2 /\left(\alpha+\beta_{0}\right)$ (this follows from the definition of $B_{n}$ via induction), so that

$$
\gamma(1)=1-\alpha=1-B_{n}\left(x_{0}+\delta\right) .
$$

Note also that

$$
\operatorname{supp} B_{n}\left(x_{0}+\delta+\cdot\right)=\left[-x_{0}-\delta,-x_{0}-\delta+n+1\right] .
$$

Thus (and once again using the symmetry and decreasing properties of $B_{n}$ around $x_{0}$ ), for any integer $N \geq-x_{0}+\delta+n+1$, we get

$$
\begin{aligned}
B(1) & =\gamma(1)+\sum_{k \neq 0} \beta_{k}=1-B_{n}\left(x_{0}+\delta\right)+2 \sum_{k=1}^{\infty} B_{n}\left(x_{0}+k-\delta\right) \\
& =A(1)+\sum_{k=1}^{N}\left(B_{n}\left(x_{0}+k-\delta\right)-B_{n}\left(x_{0}+k+\delta\right)\right) \\
& =A(1)+\sum_{k=1}^{N} \int_{\mathbb{R}}\left(\frac{\sin (\pi \xi)}{\pi \xi}\right)^{n+1}(\cos (2 \pi(k-\delta) \xi)-\cos (2 \pi(k+\delta) \xi)) \mathrm{d} \xi \\
& =A(1)+\int_{\mathbb{R}}\left(\frac{\sin (\pi \xi)}{\pi \xi}\right)^{n+1} \frac{\sin (2 \pi \xi \delta)}{\pi \xi}(\cos (\pi \xi)-\cos ((2 N+1) \pi \xi)) \mathrm{d} \xi,
\end{aligned}
$$


where the last equality follows from the fact that

$$
\begin{aligned}
& \sum_{k=1}^{N}(\cos (2 \pi \xi(k-\delta))-\cos (2 \pi \xi(k+\delta))) \\
& =2 \sum_{k=1}^{N} \sin (2 \pi \xi k) \sin (2 \pi \xi \delta)=2 \frac{\sin (2 \pi \xi \delta)}{\sin (\pi \xi)} \sum_{k=1}^{N} \sin (\pi \xi) \sin (2 \pi \xi k) \\
& =\frac{\sin (2 \pi \xi \delta)}{\sin (\pi \xi)} \sum_{k=1}^{N}(\cos (\pi \xi(2 k-1))-\cos (\pi \xi(2 k+1))) \\
& =\frac{\sin (2 \pi \xi \delta)}{\sin (\pi \xi)}(\cos (\pi \xi)-\cos ((2 N+1) \pi \xi)) .
\end{aligned}
$$

When the Parseval relation is applied to (3.53), it follows that the integral resulting from the last term vanishes, and

$$
\begin{aligned}
B(1) & =A(1)+\int_{\mathbb{R}}\left(\frac{\sin (\pi \xi)}{\pi \xi}\right)^{n+1} \frac{\sin (2 \pi \xi \delta)}{\pi \xi} \cos (\pi \xi) \mathrm{d} \xi \\
& =A(1)+\int_{\frac{1}{2}-\delta}^{\frac{1}{2}+\delta} B_{n}\left(x+\frac{n+1}{2}\right) \mathrm{d} x .
\end{aligned}
$$

Now (ii) follows from insertion of (3.54) and (3.50) in the condition $A(1) B(1)<$ 1 . Since $B_{n}$ by definition is a polynomial on every interval $[k, k+1]$ ( $k$ integer), it follows that (ii) is a polynomial inequality.

(iii): The computations in this case are very similar: In a condition $A B<1$ (and with $x_{0}=\frac{n+1}{2}$ ) we need to compute the factors

$$
A=\sup _{\sup \left|\delta_{k}\right|<\delta} \sup _{l \in \mathbb{Z}} \frac{\sum_{j \neq 0}\left|B_{n}\left(x_{0}+j+\delta_{l}\right)\right|}{\left|B_{n}\left(x_{0}+\delta_{l}\right)\right|}=\sup _{\sup \left|\delta_{k}\right|<\delta} \sup _{l \in \mathbb{Z}} \frac{1-B_{n}\left(x_{0}+\delta_{l}\right)}{B_{n}\left(x_{0}+\delta_{l}\right)}
$$

and

$$
\begin{aligned}
B & =\sup _{\sup \left|\delta_{k}\right|<\delta} \sup _{l \in \mathbb{Z}} \frac{\sum_{j \neq 0}\left|B_{n}\left(x_{0}+j+\delta_{j+l}\right)\right|}{\left|B_{n}\left(x_{0}+\delta_{l}\right)\right|}=\frac{2 \sum_{j=1}^{\infty}\left|B_{n}\left(x_{0}+j-\delta\right)\right|}{\left|B_{n}\left(x_{0}-\delta\right)\right|} \\
& =A+\frac{\sum_{k=1}^{N}\left(B_{n}\left(x_{0}+k-\delta\right)-B_{n}\left(x_{0}+k+\delta\right)\right)}{\left|B_{n}\left(x_{0}-\delta\right)\right|} \\
& =A+\frac{\int_{\mathbb{R}}\left(\frac{\sin (\pi \xi)}{\pi \xi}\right)^{n+1} \frac{\sin (2 \pi \xi \delta)}{\pi \xi} \cos (\pi \xi) \mathrm{d} \xi}{\left|B_{n}\left(x_{0}-\delta\right)\right|}=A+\frac{\int_{\frac{1}{2}-\delta}^{\frac{1}{2}+\delta} B_{n}\left(x+x_{0}\right) \mathrm{d} x}{\left|B_{n}\left(x_{0}-\delta\right)\right|},
\end{aligned}
$$

so that the inequality $A B<1$ becomes

$$
\begin{aligned}
\left(1-B_{n}\left(x+x_{0}\right)\right)\left(1-B_{n}\left(x+x_{0}\right)+\int_{\frac{1}{2}-\delta}^{\frac{1}{2}+\delta} B_{n}\left(x+x_{0}\right) \mathrm{d} x\right) & \\
& <B_{n}\left(x+x_{0}\right)^{2},
\end{aligned}
$$


which after a straightforward simplification gives condition (iii).

Example 3.1. Proposition 3.2 gives upper limits $\delta$ that we have computed numerically (in Maple) and listed in Table1. (Although some of the polynomial inequalities are easily solved exactly, the size of the bound appears more clearly from the numerical values given in the table.) These bounds are larger than those found by Sur ${ }^{20}$ with a different method, which however also give bounds for $B_{7}, B_{8}$ and the best possible bound (see Example 1.1) for the Shannon wavelet. Another approach proposed in ${ }^{5}$ gives $\delta=1 / 2$ for $B_{1}$ but only $\delta=1 / 4$ for $B_{2}$.

The upper bounds given in Table 1 are not sharp, at least not for $B_{1}$ (which sometimes is called the Franklin scaling function), which has an upper bound $\delta=\frac{1}{2}$. Liu and Walter ${ }^{14}$ showed that this bound is sharp in the sense that there is no reconstruction formula $f=\sum_{k} f\left(\lambda_{k}\right) \widetilde{q_{\lambda_{k}}}$ when $\lambda_{k}=k+1 / 2$ (that is, $x_{0}=1$ and $\left.\delta_{k}=-1 / 2\right)$. They did also show that the reconstruction formula holds for $\delta=1 / 2$ under the restriction that all $\delta_{k}$ have the same sign. Then they claimed (without proof) that this restriction can be removed. In our next theorem, we prove that this claim is correct. Moreover, our proof is different from that of Liu and Walter also for the special cases they proved. Our proof is based on the sharpened version of Theorem 3.1 that we described in Remark 3.2 .

Theorem 3.3. For the Franklin wavelet, the irregular sampling reconstruction formula $f=\sum_{k} f\left(\lambda_{k}\right) \widetilde{q_{\lambda_{k}}}$ in (3.20) holds with a sharp upper bound $\delta=1 / 2$.

Proof. The sharpness is already proved ${ }^{14}$. Set $x_{0}=1, \delta \in(0,1 / 2]$,

$$
\varphi\left(x_{0}+x\right)=B_{1}(x+1) \stackrel{\text { def }}{=}\left\{\begin{array}{ll}
1-|x| & \text { if }|x|<1, \\
0 & \text { otherwise }
\end{array} \text { and } \quad \mathrm{T} \stackrel{\text { def }}{=} \mathrm{I}-\Lambda \Phi,\right.
$$

with $\Lambda$ being a diagonal matrix with $k$ th entry $1 /\left(1-\left|\delta_{k}\right|\right)$ (as in Theorem 3.1 (iii)). It follows that $(\mathrm{T})_{k, k}=0$ and, since $\left|\delta_{l}\right|<1 / 2$, the only possible nonzero elements occur if $l-k$ and $\delta_{l}$ have opposite signs and $l-k= \pm 1$, so that

\begin{tabular}{|l|l|l|}
\hline Scaling function & $\delta$ such that $\|\mathrm{I}-\Phi\|<1$ & $\delta$ such that $\|\mathrm{I}-\Lambda \Phi\|<1$ \\
\hline \hline$B_{1}$ & .4082482905 & .4142135624 \\
\hline$B_{2}$ & .3999020374 & .4068032513 \\
\hline$B_{3}$ & .3317981368 & .3389234577 \\
\hline$B_{4}$ & .2601307648 & .2661625543 \\
\hline$B_{5}$ & .1659471664 & .1693893244 \\
\hline$B_{6}$ & .04682311225 & .04723036898 \\
\hline
\end{tabular}

Table 1. B-spline scaling function jitter error bounds computed from Proposition $3.2(\underline{\text { iii })}$ and $(\underline{i i i)}$. The listed bounds are accurate up to a possible roundoff error in the last decimal. 


$$
\begin{gathered}
(\Phi)_{k, l}=\overline{\varphi\left(x_{0}+l-k+\delta_{l}\right)}=1-\left|l-k+\delta_{l}\right|=1-\left(1-\left|\delta_{l}\right|\right)=\left|\delta_{l}\right| \text { and } \\
(\mathrm{T})_{k, l}= \begin{cases}\left|\frac{\varphi\left(x_{0}+l-k+\delta_{l}\right)}{\varphi\left(x_{0}+\delta_{k}\right)}\right|=\frac{\left|\delta_{l}\right|}{1-\left|\delta_{k}\right|} \leq \frac{|\delta|}{1-|\delta|} \stackrel{\text { def }}{=} \delta^{\prime}, & \text { if } l \neq k \text { and }\left|l-k+\delta_{l}\right|<1, \\
0 & \text { otherwise. }\end{cases}
\end{gathered}
$$

Recall from Remark 3.2 and the Schur estimate (3.28) that it is enough to find a condition on $\delta$ which guarantees that

$$
\begin{aligned}
\sup _{\sup \left|\delta_{k}\right|<\delta}\left\|\mathrm{T}^{k}\right\|_{\mathcal{B}\left(l_{2}, l_{2}\right)}^{2} & \leq \sup _{\sup \left|\delta_{k}\right|<\delta}\left\|\mathrm{T}^{k}\right\|_{\mathcal{B}\left(l_{1}, l_{1}\right)}\left\|\mathrm{T}^{k}\right\|_{\mathcal{B}\left(l_{\infty}, l_{\infty}\right)} \\
& =\sup _{\sup \left|\delta_{k}\right|<\delta} \sup \left(\sum_{i}\left|\left(\mathrm{~T}^{k}\right)_{i, j}\right|\right) \cdot \sup _{i}\left(\sum_{j}\left|\left(\mathrm{~T}^{k}\right)_{i, j}\right|\right)<1,
\end{aligned}
$$

For $k=1$, we see from (3.56) that each column contain exactly one nonzero element (either above or below the main diagonal, depending on the sign of each $\delta_{l}$ ). It follows that $\sup _{\sup \left|\delta_{k}\right|<\delta}\left\|\mathrm{T}^{k}\right\|_{\mathcal{B}\left(l_{1}, l_{1}\right)}\left\|\mathrm{T}^{k}\right\|_{\mathcal{B}\left(l_{\infty}, l_{\infty}\right)}$ is attained for some matrix $\mathrm{T}$ that consists of (finite or (half- or doubly) infinite) submatrices with the following simple structure and with the main diagonal indicated with boldface printing:

$$
\begin{aligned}
\mathrm{T}_{1} & =\left(\begin{array}{llllll}
\ddots & & & & \\
\ddots & \mathbf{0} & & & \\
& \delta^{\prime} & \mathbf{0} & & \\
& & \delta^{\prime} & \mathbf{0} & \\
& & & \delta^{\prime} & \ddots \\
& & & & \ddots
\end{array}\right), \quad \mathrm{T}_{2}=\left(\begin{array}{ccccc}
\ddots & & & & \\
\ddots & \delta^{\prime} & & & \\
& \mathbf{0} & \delta^{\prime} & & \\
& & \mathbf{0} & \delta^{\prime} & \\
& & & \mathbf{0} & \ddots \\
& & & & \ddots
\end{array}\right), \quad \text { and } \\
\mathrm{T}_{3} & =\left(\begin{array}{llll}
\delta^{\prime} & \mathbf{0} & \delta^{\prime} & 0 \\
0 & \delta^{\prime} & \mathbf{0} & \delta^{\prime}
\end{array}\right), \quad \text { where } \delta^{\prime}=\frac{\delta}{1-|\delta|} \quad \text { and } \quad \delta<1 .
\end{aligned}
$$

(The finite sub-matrix $\mathrm{T}_{3}$ appears in the intersection when the lower right corner of a matrix of type $\mathrm{T}_{1}$ touch the upper left corner of a matrix of type $\mathrm{T}_{2}$.) It is apparent from (3.57), that the largest possible $\|\mathrm{T}\|$ occurs if $i$ and $j$ in the last suprema "pinpoints" a submatrix of type $\mathrm{T}_{3}$. Similarly, for $k=2$ and $k=4$, 
$\sup _{\sup \left|\delta_{k}\right| \leq \delta^{\prime}}\left\|\mathrm{T}^{k}\right\|$ is attained for a matrix $\mathrm{T}^{k}$ with the following nonzero diagonals:

$$
\begin{aligned}
& \mathrm{T}^{2}=\left(\begin{array}{cccccccccccc}
\ddots & \ddots & \ddots & \ddots & \ddots & & & & & & & \\
& \delta^{\prime 2} & 0 & 0 & 0 & 0 & & & & & & \\
& & \delta^{\prime 2} & 0 & 0 & 0 & 0 & & & & \\
& & \delta^{\prime 2} & 0 & \boldsymbol{\delta}^{\prime 2} & 0 & \delta^{\prime 2} & & & \\
\\
& & & \delta^{\prime 2} & 0 & \boldsymbol{\delta}^{\prime 2} & 0 & \delta^{\prime 2} & & \\
& & & & & 0 & 0 & \mathbf{0} & 0 & \delta^{\prime 2} & \\
& & & & & & 0 & 0 & \mathbf{0} & 0 & \delta^{\prime 2} \\
& & & & & & & \ddots & \ddots & \ddots & \ddots & \ddots
\end{array}\right) \quad \text { and } \\
& \mathrm{T}^{4}=\left(\begin{array}{cccccccccccccc}
\ddots & \ddots & \ddots & \ddots & \ddots & \ddots & \ddots & \ddots & \ddots & & & & \\
& \delta^{\prime 4} & 0 & 0 & 0 & \mathbf{0} & 0 & 0 & 0 & 0 & & & \\
& \delta^{\prime 4} & 0 & \delta^{\prime 4} & 0 & \boldsymbol{\delta}^{\prime 4} & 0 & \delta^{\prime 4} & 0 & \delta^{\prime 4} & & \\
& & \delta^{\prime 4} & 0 & \delta^{\prime 4} & 0 & \boldsymbol{\delta}^{\prime 4} & 0 & \delta^{\prime 4} & 0 & \delta^{\prime 4} & \\
& & 0 & 0 & 0 & 0 & 0 & \mathbf{0} & 0 & 0 & 0 & \delta^{\prime 4} \\
& & & \ddots & \ddots & \ddots & \ddots & \ddots & \ddots & \ddots & \ddots & \ddots & \ddots
\end{array}\right) .
\end{aligned}
$$

Via induction and (3.57), we get

$$
\left\|\mathrm{T}^{2^{k}}\right\|_{\mathcal{B}\left(l_{2}, l_{2}\right)} \leq\left\|\mathrm{T}^{2^{k}}\right\|_{\mathcal{B}\left(l_{1}, l_{1}\right)} \cdot\left\|\mathrm{T}^{2^{k}}\right\|_{\mathcal{B}\left(l_{\infty}, l_{\infty}\right)}=\delta^{\prime 2^{k}} \cdot\left(2^{k}+1\right) \delta^{\prime 2^{k}}
$$

Hence $\left\|\mathrm{T}^{2^{k}}\right\|_{\mathcal{B}\left(l_{2}, l_{2}\right)}<1$ if

$$
\delta^{\prime}<\left(2^{k}+1\right)^{-\frac{1}{2 \cdot 2^{k}}}=\left(\left(2^{k}+1\right)^{\frac{1}{2^{k}+1}}\right)^{-\frac{2^{k}+1}{2 \cdot 2^{k}}} \nearrow 1, \quad \text { as } k \rightarrow \infty
$$

where $\nearrow$ denotes convergence from below. In other words, for any $\delta \stackrel{\text { def }}{=} \sup _{l}\left|\delta_{l}\right|<$ $1 / 2$ (and thus also for $\delta=1 / 2$ ), it follows that $\delta^{\prime}<1$ and, for some $k \in \mathbb{Z}$, $\left\|\mathrm{T}^{2^{k}}\right\|_{\mathcal{B}\left(l_{2}, l_{2}\right)}<1$ so that $\Phi \in \mathcal{G}\left(l_{2}\right)$ (and $\Phi^{-1}$ can be computed, e.g., with a Neumann series expansion). This concludes our proof.

\section{Acknowledgments}

We would like to thank the referees for suggestions that helped us simplify the presentation and Professor Lubomir T. Dechevsky for insightful discussions and suggestions. During the final revisions of this paper, the second named author was supported by the Swedish Research Council, postdoc fellowship no. 623-2003-105.

\section{References}

1. A. Aldroubi and K. Gröchenig. Beurling-Landau-type theorems for non-uniform sampling in shift invariant spline spaces. J. Fourier Anal. Appl., 6(1):93-103, 2000.

2. A. Aldroubi and K. Gröchenig. Nonuniform sampling and reconstruction in shiftinvariant spaces. SIAM Rev., 43(4):585-620, 2001. 
3. J. Bergh and J. Löfström. Interpolation Spaces, volume 223 of Die Grundlehren der Mathematischen Wissenschaften. Springer, Berlin, 1976.

4. W. Chen, S. Itoh, and J. Shiki. Irregular sampling theorems for wavelet subspaces. IEEE Trans. Inform. Theory, 44(3):1131-1142, May 1998.

5. W. Chen, S. Itoh, and J. Shiki. On sampling in shift invariant spaces. IEEE Trans. Inform. Theory, 48(10):2802-2810, 2002. WWW: http://wWw.math.ualberta.ca/ $\sim$ wenchen/files/publications.html.

6. S. Ericsson and N. Grip. Efficient wavelet prefilters with optimal time-shifts. IEEE Trans. Signal Process., (to appear). WWW: http://www.sm.luth.se/ grip/Resear ch/publications.html

7. H. Feichtinger and K. Gröchenig. Theory and practice of irregular sampling. In J. J. Benedetto and M. W. Frazier, editors, Wavelets: Mathematics and Applications, chapter 8, pages 305-363. CRC Press, Boca Raton, FL, 1994.

8. N. Grip. Wavelet and Gabor Frames and Bases: Approximation, Sampling and Applications. Doctoral thesis 2002:49, Luleå University of Technology, SE-971 87 Luleå, 2002. WWW: http://www.sm.luth.se/ grip/Research/publications.html.

9. N. Grip and W. Sun. Remarks on the article "On the stability of wavelet and Gabor frames (Riesz bases)" by J. Zhang. J. Fourier Anal. Appl., 9(1):97-100, 2003.

10. K. Gröchenig. Localization of frames, Banach frames, and the invertibility of the frame operator. J. Fourier Anal. Appl., 10(2), 2004.

11. E. Hernández and G. Weiss. A First Course on Wavelets. CRC Press, 1996.

12. A. J. E. M. Janssen. The Zak transform and sampling theorems for wavelet subspaces. IEEE Trans. Signal Process., 41(12):3360-3364, Dec. 1993.

13. Y. Katznelson. An Introduction to Harmonic Analysis. Dover Publications, Inc, second corrected edition, 1976.

14. Y. Liu and G. G. Walter. Irregular sampling in wavelet subspaces. J. Fourier Anal. Appl., 2(2):181-189, 1995.

15. W. Qiao. Regular and irregular sampling theorems of Shannon's type in wavelet subspace. Math. Appl., 11(3):90-94, 1998.

16. W. Rudin. Real and Complex Analysis. McGraw-Hill, third edition, 1987.

17. W. Rudin. Functional Analysis. McGraw Hill, second edition, 1991.

18. J. Schur. Bemerkungen zur Theorie der beschränkten Bilinearformen mit unendlich vielen Veränderlichen. J. Reine Angew. Math., 140(1):1-28, 1911.

19. G. Strang. Linear Algebra and its applications. Harcourt Brace Jovanovich College publishers, third edition, 1988. Library of Congress Catalog Card Number: 87-083019.

20. W. Sun. Sampling theorems for multivariate shift invariant subspaces. 2003. Preprint.

21. W. Sun and X. Zhou. On Kadec's 1/4-theorem and the stability of Gabor frames. Appl. Comput. Harmon. Anal., 7(2):239-242, Sept. 1999.

22. W. Sun and X. Zhou. Sampling theorem for wavelet subspaces: Error estimate and irregular sampling. IEEE Trans. Signal Process., 48(1):223-226, 2000.

23. G. G. Walter. A sampling theorem for wavelet subspaces. IEEE Trans. Inform. Theory, 38(2):881-884, Mar. 1992.

24. G. G. Walter. Non-uniform sampling in wavelet subspaces. In 1999 IEEE International Conference on Acoustics, Speech and Signal Processing (ICASSP '99): proceedings, volume 4, Phoenix, AZ, USA, Mar. 1999.

25. R. M. Young. An Introduction to Nonharmonic Fourier Series and Wavelet Expansions. Academic Press, revised first edition, Apr. 2001.

26. X. Zhou and W. Sun. On the sampling theorem for wavelet subspaces. J. Fourier Anal. Appl., 5(4):347-354, 1999. 